\title{
COMPARAÇÃO ENTRE MÉTODOS DE SÍNTESE DE ORTOSILICATO DE LÍTIO NA REAÇÃO DE CARBONATAÇÃ̃O.
}

\author{
A. ASSAD FILHO e T.L.P. DANTAS ${ }^{1}$ \\ ${ }^{1}$ Universidade Federal do Paraná, Departamento de Engenharia Química \\ E-mail para contato: tirzha@ufpr.br
}

\begin{abstract}
RESUMO - Sabe-se que os combustíveis fósseis ainda são a forma dominante de fonte energética no mundo; e, consequentemente, dentre as implicações ambientais deste uso, pode-se destacar o aumento da concentração do dióxido de carbono no ar atmosférico. Alguns estudos sobre a sorção de dióxido de carbono em compostos a base de lítio têm mostrado que este tipo de material é promissor para captura de $\mathrm{CO}_{2}$ a partir de gases a elevada temperatura e que a reação é reversível na faixa de temperatura de $100^{\circ} \mathrm{C}$ a $900^{\circ} \mathrm{C}$. O objetivo deste trabalho é sintetizar ortosilicato de lítio de maneiras distintas e realizar a captura de dióxido de carbono através da reação de carbonatação com os sólidos sintetizados. As amostras de $\mathrm{Li}_{4} \mathrm{SiO}_{4}$ foram sintetizadas, a $100^{\circ} \mathrm{C}$, a partir da reação do carbonato de lítio com óxido de silício dissolvido com hidróxido de sódio ou com hidróxido de lítio com óxido de silício. A reação de carbonatação dos sólidos, a $500^{\circ} \mathrm{C}$, foi avaliada durante 1 (uma) hora fazendo passar uma mistura $0,3 \% \mathrm{CO}_{2} / \mathrm{He}$ em um leito fixo. $\mathrm{Na}$ saída do leito, a concentração de dióxido de carbono era medida por cromatografia gasosa. Constatouse que os sólidos sintetizados são competitivos para serem utilizados na captura de dióxido de carbono.
\end{abstract}

\section{INTRODUÇÃO}

Existem várias tecnologias que podem ser aplicadas para a remoção de $\mathrm{CO}_{2}$; no entanto, muitas tecnologias ainda possuem limitações econômicas e operacionais. Algumas das opções atualmente identificadas para a separação e posterior captura do dióxido de carbono incluem as seguintes tecnologias: absorção, separação por membranas, fixação do carbono e sorção (adsorção física ou química).

Um dos fatores limitantes para o uso destas tecnologias é a alta temperatura na qual o $\mathrm{CO}_{2}$ é gerado. Portanto, o desenvolvimento de tecnologias para remoção desse produto em altas temperaturas é desejável.

A técnica de separação de $\mathrm{CO}_{2}$ em altas temperaturas através da reação reversível com um sólido inorgânico é uma das formas mais promissoras de capturar $\mathrm{CO}_{2}$. Os compostos a base de lítio tem recebido especial atenção devido a sua capacidade de reagir reversivelmente com $\mathrm{CO}_{2}$ em uma ampla faixa de temperatura (Nagawaka e Ohashi, 1999; Gauer, 2006; Venegas et al., 2007; Nair et al., 2009). Os compostos a base de lítio mais estudados são o óxido de lítio ( $\left.\mathrm{Li}_{2} \mathrm{O}\right)$, o zirconato de lítio $\left(\mathrm{Li}_{2} \mathrm{ZrO}_{3}\right)$, os silicatos de lítio $\left(\mathrm{Li}_{4} \mathrm{SiO}_{4}\right.$ e $\left.\mathrm{Li}_{2} \mathrm{SiO}_{3}\right)$, o aluminato de lítio $\left(\mathrm{Li}_{5} \mathrm{AlO}_{4}\right)$, a ferrita de lítio $\left(\mathrm{LiFeO}_{2}\right)$ e o titanato de lítio $\left(\mathrm{Li}_{4} \mathrm{TiO}_{4}\right)($ Ortiz-Landeros et al., 2012). 
$\mathrm{O} \mathrm{Li}_{2} \mathrm{O}$ e o $\mathrm{Li}_{5} \mathrm{AlO}_{4}$ se destacam pela suas altas capacidade para a sorção de $\mathrm{CO}_{2}$ se comparado com os outros compostos de lítio. Porém, as características de alta reatividade e corrosividade do $\mathrm{Li}_{2} \mathrm{O}$ tornam seu emprego desvantajoso. Já o $\mathrm{Li}_{5} \mathrm{AlO}_{4}$ sofre o efeito de sinterização com o aumento da temperatura. A sinterização é responsável por diminuir a área superficial e, consequentemente, diminuir a capacidade de reação do sólido (Ávalos-Rendón et al., 2009).

$\mathrm{O} \mathrm{Li}_{4} \mathrm{TiO}_{4}$, o $\mathrm{LiFeO}_{2}$ e o $\mathrm{Li}_{2} \mathrm{ZrO}_{3}$ possuem as menores capacidades teóricas de sorção de dióxido de carbono. Nakagawa et al. (2002) reportaram que a temperatura de equilíbrio da reação do $\mathrm{LiFeO}_{2}$ é relativamente baixa $\left(510^{\circ} \mathrm{C}\right)$ se comparada com a temperatura de equilíbrio do $\mathrm{Li}_{2} \mathrm{ZrO}_{3}\left(715^{\circ} \mathrm{C}\right)$ e do $\mathrm{Li}_{4} \mathrm{SiO}_{4}\left(720^{\circ} \mathrm{C}\right)$. Já a alta capacidade do $\mathrm{Li}_{2} \mathrm{SiO}_{3}$ não o torna competitivo para captura em altas temperaturas, pois a temperatura de equilíbrio da reação com $\mathrm{CO}_{2}$ é de $260^{\circ} \mathrm{C}$ (Nakagawa et al., 2003).

Ida e Lin (2003) estudaram o mecanismo de sorção de dióxido de carbono em zirconato de lítio $\left(\mathrm{Li}_{2} \mathrm{ZrO}_{3}\right)$ e mostraram que este tipo de material é promissor para captura de $\mathrm{CO}_{2}$ a partir de gases a elevada temperatura. A reação mostrou-se reversível na faixa de temperatura de $450^{\circ} \mathrm{C}$ a $900^{\circ} \mathrm{C}$. Eles observaram que a $500^{\circ} \mathrm{C}$ esta é uma reação muito lenta, apesar de a capacidade para sorção de $\mathrm{CO}_{2}$ ser bastante elevada. Nagawaka e Ohashi (1999) também relataram que a adição de carbonato de lítio e de carbonato de potássio ao $\mathrm{Li}_{2} \mathrm{ZrO}_{3}$ aumenta a velocidade de sorção; mas o mecanismo do processo de sorção ainda não é completamente compreendido.

Kato et al. (2002) testaram vários sólidos baseados em lítio e identificaram que o ortosilicato de lítio $\left(\mathrm{Li}_{4} \mathrm{SiO}_{4}\right)$ apresenta uma cinética de carbonatação, a $500^{\circ} \mathrm{C}$, trinta vezes mais rápida que a do zirconato de lítio. Os autores também constataram que esse sólido possui uma cinética mais rápida que a do metasilicato de lítio $\left(\mathrm{Li}_{2} \mathrm{SiO}_{3}\right)$ a temperatura ambiente (Kato et al., 2005).

Uma comparação entre $\mathrm{Li}_{2} \mathrm{ZrO}_{3}$ e $\mathrm{Li}_{4} \mathrm{SiO}_{4}$ pode ser feita através das suas reações de equilíbrio (Nagawaka et al., 2002); Equações (1) e (2).

$$
\begin{aligned}
& \mathrm{Li}_{2} \mathrm{ZrO}_{3}+\mathrm{CO}_{2} \leftrightarrow \mathrm{Li}_{2} \mathrm{CO}_{3}+\mathrm{ZrO}_{2} \\
& \mathrm{Li}_{4} \mathrm{SiO}_{4}+\mathrm{CO}_{2} \leftrightarrow \mathrm{Li}_{2} \mathrm{CO}_{3}+\mathrm{Li}_{2} \mathrm{SiO}_{3}
\end{aligned}
$$

Comparando-se as reações (Equação (1) e Equação (2)), existe uma aparente desvantagem com relação ao ortosilicato de lítio já que somente dois átomos seus participam do processo. No entanto, a sua capacidade de sorção é $28 \%$ maior se comparada com o $\mathrm{Li}_{2} \mathrm{ZrO}_{3}$. Além do que; no caso do $\mathrm{Li}_{4} \mathrm{SiO}_{4}$, a matéria prima envolvida é bem mais barata (Kato et al., 2005). A quantidade máxima teórica de captura de dióxido de carbono é de um mol de $\mathrm{CO}_{2}$ para um mol de $\mathrm{Li}_{4} \mathrm{SiO}_{4}$, o que corresponde a uma capacidade máxima teórica de 36,7\% em massa. Na faixa de temperatura em que ocorre a reação do $\mathrm{Li}_{4} \mathrm{SiO}_{4}$ com o $\mathrm{CO}_{2}$, não é provável a reação do produto $\mathrm{Li}_{2} \mathrm{SiO}_{3}$ com o $\mathrm{CO}_{2}$ presente na atmosfera devido à baixa temperatura de equilíbrio (Ortiz-Landeros et al., 2011).

Do ponto de vista cinético, a reação representada pela equação (2) ocorre em duas etapas (Pannocchia et al., 2007). Primeiro, o ortosilicato de lítio se decompõe de acordo com a seguinte reação; Equação (3):

$$
\mathrm{Li}_{4} \mathrm{SiO}_{4} \rightarrow 2 \mathrm{Li}^{+}+\mathrm{O}^{2-}+\mathrm{Li}_{2} \mathrm{SiO}_{3}
$$


Então o $\mathrm{CO}_{2}$ reage com os íons de lítio e oxigênio para produzir o $\mathrm{Li}_{2} \mathrm{CO}_{3}$; Equação (4):

$$
2 \mathrm{Li}^{+}+\mathrm{O}^{2+}+\mathrm{CO}_{2} \rightarrow \mathrm{Li}_{2} \mathrm{CO}_{3}
$$

Pfeiffer et al. (1998) estudaram diferentes métodos de síntese do $\mathrm{Li}_{4} \mathrm{SiO}_{4}$, sendo o método de síntese no estado sólido o que apresentou melhor resultado. Bretado et al. (2005) sintetizaram $\mathrm{Li}_{4} \mathrm{SiO}_{4}$ a partir de diferentes métodos; e, a partir da caracterização, relataram que a temperatura de síntese está associada à capacidade para sorção de $\mathrm{CO}_{2}$. Diferentes materiais precursores foram testados por Seggiani et al. (2011) para sintetizar $\mathrm{Li}_{4} \mathrm{SiO}_{4}$; como resultado os autores obtiveram que diferentes tipos de sílica $\left(\mathrm{SiO}_{2}\right)$ não afetam a capacidade de carbonatação do ortosilicato de lítio. No entanto, os autores confirmaram que a adição de $\mathrm{K}_{2} \mathrm{CO}_{3}$ no ortosilicato de lítio aumenta em $80 \%$ a capacidade de captura devido à formação de uma camada de carbonato de lítio fundida na temperatura de $580^{\circ} \mathrm{C}$. Amorim (2013) também relatou que a temperatura de pré-tratamento influencia no equilíbrio da reação de carbonatação já que há diminuição da quantidade de sítios ativos pela reação de decomposição do ortosilicato de lítio em metasilicato de lítio.

Reconhecendo-se que a literatura acerca do tema ainda é bastante escassa e incipiente, este trabalho tem como objetivo sintetizar ortosilicato de lítio de maneiras distintas e realizar a captura de dióxido de carbono através da reação de carbonatação com os sólidos sintetizados.

\section{MATERIAIS E METODOLOGIA.}

\subsection{Reagentes}

Todos os reagentes de lítio - carbonato de lítio e hidróxido de lítio - foram gentilmente cedidos pela Chemetall (Alemanha). Todos estes sólidos eram de pureza mínima de 98,5\%. Utilizou-se óxido de silício no formato sílica gel branca (1 a $4 \mathrm{mmm}$ ) da marca Vetec (Brasil). Todos os demais reagentes eram de pureza analítica.

\subsection{Síntese dos sólidos}

Os sólidos ortosilicato de lítio foram sintetizados através de dois métodos diferentes baseados em metodologia disponível na literatura (Betrado et al., 2005).

O primeiro sólido, nomeado ortosilicato-CSi, foi sintetizado a partir de carbonato de lítio $\left(\mathrm{Li}_{2} \mathrm{CO}_{3}\right)$ e óxido de silício $\left(\mathrm{SiO}_{2}\right)$ diluído em solução de hidróxido de sódio $(\mathrm{NaOH})$. Primeiramente, foram dissolvidos $60 \mathrm{~g}$ de $\mathrm{SiO}_{2}$ em $200 \mathrm{~mL}$ de uma solução $10 \mathrm{~mol} / \mathrm{L}$ de $\mathrm{NaOH}$ mantida a $55^{\circ} \mathrm{C}$ e agitação constante por 40 minutos. Após, foram acrescentados à solução $148 \mathrm{~g}$ de $\mathrm{Li}_{2} \mathrm{CO}_{3}$. A mistura foi então mantida sob agitação constante por 90 minutos e a temperatura de $100^{\circ} \mathrm{C}$ até que se observou que toda a água evaporou. As reações a seguir representam a sequência da síntese; Equações (5) a (7):

$$
\begin{aligned}
& \mathrm{SiO}_{2}+2 \mathrm{NaOH} \rightarrow \mathrm{Na}_{2} \mathrm{SiO}_{3}+\mathrm{H}_{2} \mathrm{O} \\
& \mathrm{Na}_{2} \mathrm{SiO}_{3}+\mathrm{Li}_{2} \mathrm{CO}_{3} \rightarrow \mathrm{Li}_{2} \mathrm{SiO}_{3}+\mathrm{Na}_{2} \mathrm{CO}_{3} \\
& \mathrm{Li}_{2} \mathrm{SiO}_{3}+\mathrm{Li}_{2} \mathrm{CO}_{3} \rightarrow \mathrm{Li}_{4} \mathrm{SiO}_{4}+\mathrm{CO}_{2}
\end{aligned}
$$


A reação representada pela equação (6) tem como produto o silicato de lítio. Para que haja a formação do ortosilicato desejado, deve-se reagir então o silicato de sódio formado com carbonato de lítio. Sendo assim, ao produto da reação representada pela equação (5) foi adicionada uma massa de carbonato de lítio necessária para a formação do ortosilicato de lítio.

O segundo sólido, nomeado Ortosilicato-HidroxiSi, foi sintetizado a partir de carbonato de lítio $\left(\mathrm{Li}_{2} \mathrm{CO}_{3}\right)$ e óxido de silício $\left(\mathrm{SiO}_{2}\right)$ diluído em solução de hidróxido de lítio (LiOH). Primeiramente, foram dissolvidos $60 \mathrm{~g}$ de $\mathrm{SiO}_{2}$ em $15 \mathrm{~mL}$ de uma solução $1 \mathrm{~mol} / \mathrm{L}$ de $\mathrm{LiOH}$ mantida a $70^{\circ} \mathrm{C}$ e agitação constante por 40 minutos. Após, foram acrescentados à solução $76 \mathrm{~g}$ de $\mathrm{Li}_{2} \mathrm{CO}_{3}$. A mistura foi então mantida sob agitação constante por 90 minutos e a temperatura de $100^{\circ} \mathrm{C}$. As reações a seguir representam a sequência da síntese; Eq. (8) e (9):

$$
\begin{aligned}
& \mathrm{SiO}_{2}+2 \mathrm{LiOH} \rightarrow 2 \mathrm{Li}_{2} \mathrm{SiO}_{3}+\mathrm{H}_{2} \mathrm{O} \\
& \mathrm{Li}_{2} \mathrm{SiO}_{3}+\mathrm{Li}_{2} \mathrm{CO}_{3} \rightarrow \mathrm{Li}_{4} \mathrm{SiO}_{4}+\mathrm{CO}_{2}
\end{aligned}
$$

\subsection{Análises de XPS}

Análises de XPS foram realizadas em espectrômetro MULTILAB ESCA3000 (VG Microtech, Reino Unido). A energia através do analisador era de 58,7 eV para obtenção de espectros de alta resolução. Concentrações relativas de elementos da superfície do adsorvente foram calculadas pelas medidas das áreas dos picos no espectro de alta resolução.

\subsection{Sorção de Dióxido de carbono (Reação de Carbonatação)}

Segundo Amorim (2013), a relação estequiométrica $\mathrm{CO}_{2} / \mathrm{Li}_{4} \mathrm{SiO}_{4}$ decresce com o aumento da temperatura. Na faixa de temperatura de $25^{\circ} \mathrm{C}$ a $262^{\circ} \mathrm{C}$, a cinética da reação é bastante lenta. Já em temperaturas superiores a $724^{\circ} \mathrm{C}$ ocorre reação de descarbonatação. Escolheu-se, assim, a temperatura de $500^{\circ} \mathrm{C}$ para se realizar as reações de carbonatação. Nesta temperatura, a cinética da reação é mais rápida e o equilíbrio termodinâmico é dado conforme a reação representada pela equação (2). Ainda segundo Amorim (2013), a análise de decomposição térmica do ortosilicato de lítio mostrou que o sólido perde $8,68 \%$ de sua massa, relacionadas a água, na faixa de temperatura de $25-500^{\circ} \mathrm{C}$.

A capacidade de sorção para dióxido de carbono dos sólidos, ou a reação de carbonatação, foi avaliada passando-se uma mistura padrão de 3000 ppm de dióxido de carbono - balanceado em hélio (inerte) - a uma vazão total de $30 \mathrm{~mL} \cdot \mathrm{min}^{-}{ }^{1}$ e com fluxo descendente através de um leito fixo com temperatura controlada de $500^{\circ} \mathrm{C}$. Os sólidos foram pré-tratados passando-se hélio puro a uma vazão de $100 \mathrm{~mL} \cdot \mathrm{min}^{-1}$ durante 1 hora e a $150^{\circ} \mathrm{C}$. O leito possuía uma altura de $2,5 \mathrm{~cm} \mathrm{e}$ diâmetro de 3,2 cm. A massa de Ortosilicato-CSi utilizada foi de 17,29 g e a massa de Ortosilicato-HidroxiSi foi de $28,74 \mathrm{~g}$. O aparato experimental utilizado consistiu em uma coluna tubular (Parr Instrument, EUA) fabricada em aço inoxidável T316SS. O aquecimento do sistema foi realizado em forno com temperatura interna controlada através de um termopar tipo $\mathrm{K}$. $\mathrm{O}$ sistema foi considerado adiabático: a coluna e o forno se encontravam isolados com uma camada de $20 \mathrm{~cm}$ de fibra de vidro e material refratário. A vazão de gás era medida por controladores de vazão modelo SLA5850S (Brooks® Instrument, EUA). Todos os experimentos foram realizados na pressão atmosférica. Na saída da coluna, a quantidade de dióxido de carbono presente no gás era determinada por cromatografia gasosa, utilizando o cromatógrafo gasoso CG450 (Bruker 
Chemical Analysis, Holanda) equipado com um detector de ionização de chama (FID) seguido de um metanador. A combinação metanador/FID foi utilizada por medir baixas concentrações de dióxido de carbono. A coluna utilizada no CG para análise do gás foi uma coluna Porapak-Q (Cromacon, Brasil) utilizando hidrogênio como gás de arraste. A reação de carbonatação nos sólidos foi acompanha por até 1 hora (60 minutos). A Figura 1 representa um esquema da instalação experimental utilizado para medir a capacidade de sorção para dióxido de carbono dos sólidos.

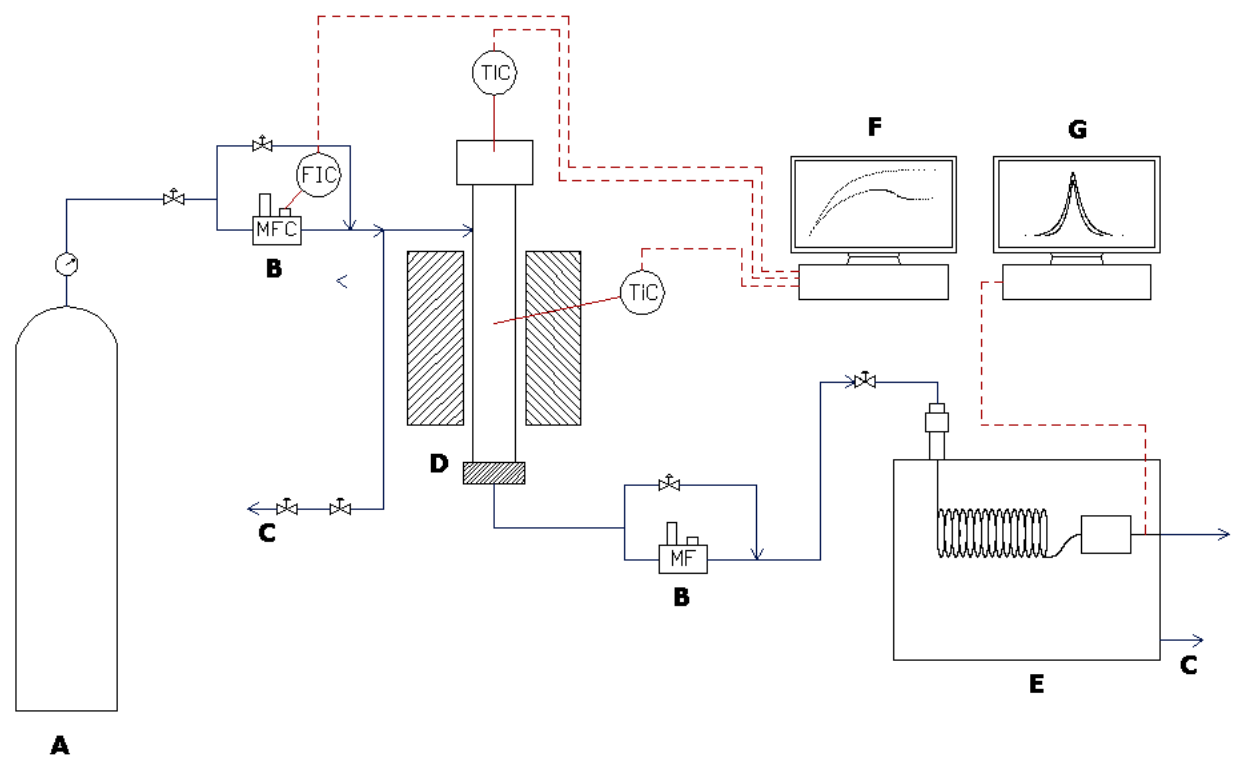

Figura 1 - Esquema da instalação experimental de sorção de dióxido (A - cilindro de mistura padrão; B - medidor e controlador de vazão; $\mathrm{C}$ - purga; D - coluna de sorção; E cromatógrafo a gás; $\mathrm{F} / \mathrm{G}$ - microcomputador).

\section{RESULTADOS E DISCUSSÃO.}

Os difratogramas de raios- $\mathrm{X}$ dos sólidos sintetizados foram compatíveis como difratograma obtido para o sólido comercialmente disponível. As análises elementares de $\mathrm{Li}, \mathrm{C}, \mathrm{O}$ e $\mathrm{Si}$ dos sólidos sintetizados são mostradas na Tabela 1. As quantidades de lítio presentes nos sólidos Ortosilicato-CSi e Ortosilicato-HidroxiSi são bastante próximas; apesar de inferiores àquelas presentes em um sólido comercialmente disponível (Dantas et al., 2014). No entanto, as quantidades relativas de $\mathrm{Li} / \mathrm{C}$ e $\mathrm{Li} / \mathrm{O}$ presentes nos sólidos sintetizados são muito similares às quantidades relativas presentes no sólido comercialmente disponível. Os resultados para o $\mathrm{Li}_{4} \mathrm{SiO}_{4}$ comercialmente disponível foram previamente reportados (Amorim, 2013; Dantas et al., 2014). Todas estas observações levam-se a crer que de fato houve a formação do ortosilicato de lítio.

A reação de carbonatação nos sólidos sintetizados foi acompanha por 1 hora (60 minutos) monitorando-se a quantidade de dióxido de carbono que deixava o leito em função do tempo. Ao longo de todos os experimentos; picos de outras substâncias foram observados, mas não puderam ser identificados por falta de padrão. No entanto, isto corrobora a teoria de que dióxido de carbono está reagindo na superfície dos sólidos. A Figura 2 representa os resultados obtidos relacionando a porcentagem de dióxido de carbono reagido versus o tempo para os sólidos sintetizados ortosilicato-CSi e ortosilicato-HidroxiSi. 
Tabela 1. Análise elementar para dos sólidos comercial e sintetizado.

\section{Elemento}

$\mathbf{L i}$

C

$\mathbf{O}$

$\mathbf{S i}$

$\mathrm{Li} / \mathrm{C}$

$\mathrm{Li} / \mathbf{O}$

\section{Ortosilicato-CSi}

22,48

17,80

48,99

10,83

1,26

0,46

\section{Ortosilicato-HidroxiSi}

Nos resultados experimentais obtidos; observa-se que, para o ortosilicato-CSi, a capacidade de carbonatação média é de 90,86\%. Já para o ortosilicato-HidroxiSi, a capacidade de carbonatação média é superior a 94\%. As duas amostras sintetizadas foram obtidas através de uma reação em que carbonato de lítio está presente. Resultados previamente reportados mostraram que a capacidade de carbonatação de um ortosilicato comercial é inferior a capacidade dos sólidos sintetizados via carbonato de lítio (Dantas et al., 2014). Segundo Nagawaka e Ohashi (1999), adição de carbonato de lítio ao zirconato de lítio aumenta a velocidade de sorção; portanto, a síntese de ortosilicato de lítio via carbonato de lítio pode ter aumentado a capacidade de carbonatação do ortosilicato. A mais baixa capacidade de carbonatação do ortosilicato-CSi pode estar relacionada a maior quantidade de impurezas já que este sólido foi obtido através de uma reação a 3 (três) etapas.

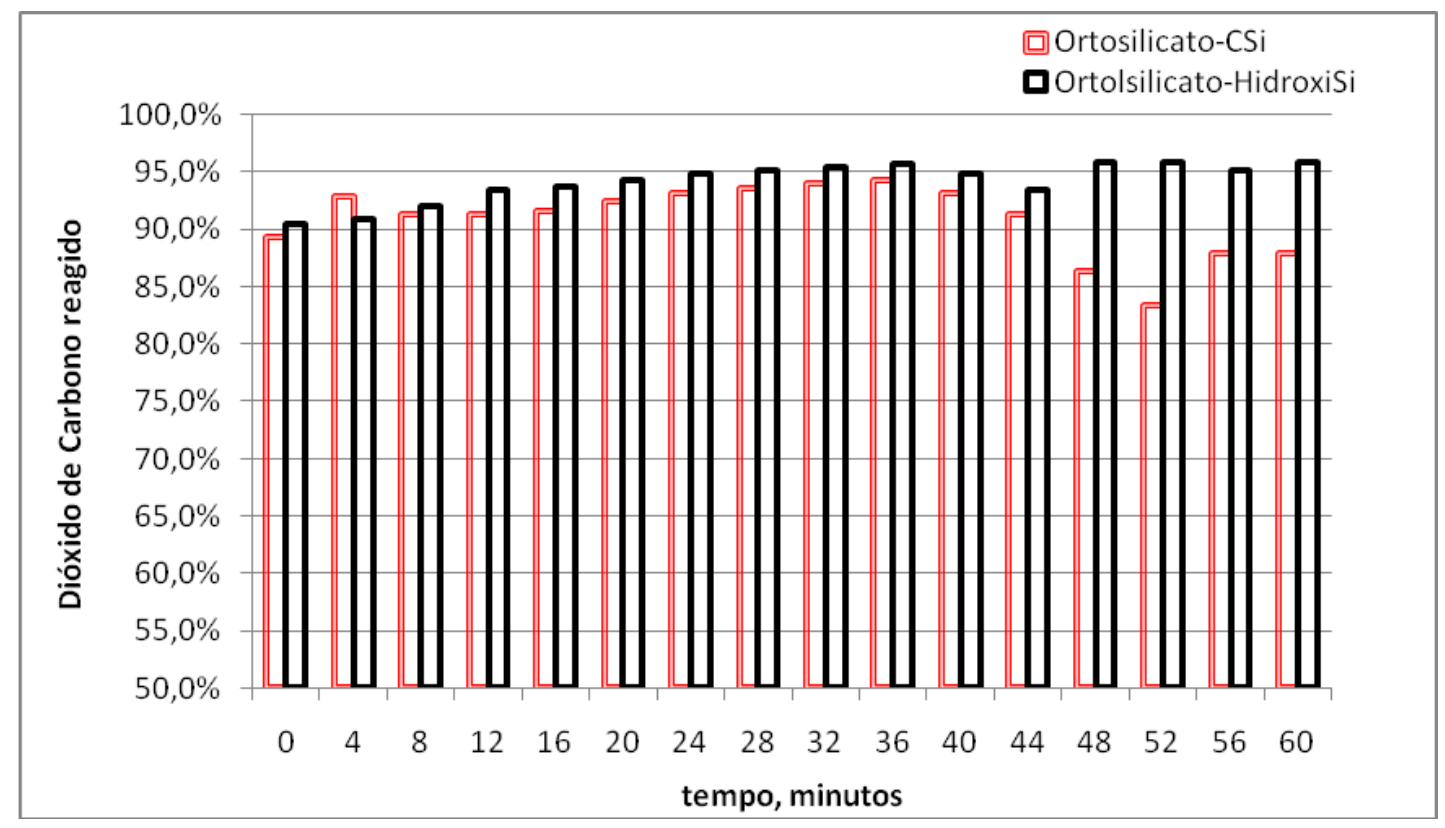

Figura 2. Porcentagem de dióxido de carbono reagido versus o tempo para os ortosilicatos sintetizados. 


\section{CONCLUSÕES}

Este é um trabalho de investigação ainda bastante incipiente mas promissor. A literatura acerca do tema é escassa e até um pouco controversa em relação, principalmente, aos métodos de síntese.

Neste trabalho comprovou-se a grande capacidade de captura do ortosilicato de lítio para o dióxido de carbono e observou-se que esta capacidade de sorção está intimamente ligada ao método de síntese. Os resultados mostraram que alta capacidade de captura de $\mathrm{CO}_{2}$ torna o ortosilicato de lítio um sólido competitivo para ser utilizado na captura de dióxido de carbono em altas temperaturas.

\section{REFERÊNCIAS}

AMORIM, S.M. Captura de $\mathrm{CO}_{2}$ em altas temperaturas por meio da reação de carbonatação do ortosilicato de lítio $\left(\mathrm{Li}_{4} \mathrm{SiO}_{4}\right)$. Dissertação (mestrado) - Universidade Federal de Santa Catarina, Programa de Pós-graduação em Engenharia Química, Florianópolis, 2013.

ÁVALOS-RENDÓN, T.; CASA-MADRID, J.;PFEIFFER, H.. Thermochemical capture of carbon dioxide on lithium aluminates $\left(\mathrm{LiAlO}_{2}\right.$ and $\left.\mathrm{Li}_{5} \mathrm{AlO}_{4}\right)$ : A new option for the $\mathrm{CO}_{2}$ absorption. J. Phys. Chem. A, v. 113, p.6919-6923, 2009.

BRETADO, M.E.; VELDERRAIN, V.G.; GUTIERREZ, D.L.; COLLINS-MARTINEZ, V.; ORTIZ, A.L. A new synthesis route to $\mathrm{Li}_{4} \mathrm{SiO}_{4}$ as $\mathrm{CO}$ catalytic/sorbent. Catal. Today, 107108, p. 863-867, 2005.

DANTAS, T.L.P.; SCHREINER, W. ASSAD FILHO; A.. Síntese de ortosilicato de lítio: Sorção de $\mathrm{CO}_{2}$ em $\mathrm{Li}_{4} \mathrm{SiO}_{4}$. Anais do X Encontro brasileiro sobre adsorção, Guarujá-SP, 2014.

GAUER, C.; HESCHEL, W. Doped lithium orthosilicate for absorption of carbon dioxide.J. Mater. Sci., v. 41, p.2405-2409. 2006.

IDA, J-I.; LIN, Y.S. Mechanism of High-Temperature $\mathrm{CO}_{2}$ Sorption on Lithium Zirconate. Environ. Sci. Technol., 37, p. 1999-2004, 2003. 
KATO, M.; NAKAGAWA, K.; ESSAKI, K.; MAEZAWA, Y.; TAKEDA, S.; KOGO, S.; HAGIWARA, Y. Novel $\mathrm{CO}_{2}$ Absorbents Using Lithium-Containing Oxide. Int. J. Appl.Ceram. Technol., 2, p. 467-475, 2005.

KATO, M.; YOSHIKAWA, S.; NAKAGAWA, K..Carbon dioxide absorption by lithium orthosilicate in a wide range of temperature and carbon dioxide concentrations. J. Mat. Sci., v. 21, p.485-487, 2002.

NAIR, B. N.; BURWOOD, V. J.; GOH, V. J.; NAKAGAWA, K.; YAMAGUCHI, T. Lithium based ceramic materials and membranes for high temperature $\mathrm{CO}_{2}$ separation. Prog. Mat. Sci., v. 54, p. 511-541. 2009.

NAKAGAWA, K.; OHASHI, T. A Reversible Change between Lithium Zirconate and Zirconia in Molten Carbonate. Electrochemistry, 67, p. 618-621, 1999.

NAKAGAWA, K.; OHASHI, T.; MASAHIRO, K.; YOSHIKAWA, S.; ESSAKI, K. US Patent No. 6387845B1, assigned to Kabushiki Kaisha Toshiba, 2002.

NAKAGAWA, K.; KATO, M.; YOSHIKAWA, S.; ESSAKI, K.; UEMOTO, H. A novel $\mathrm{CO}_{2}$ absorbent using lithium-containing oxides. Proceedings of the 2nd Annual Conference on Carbon Sequestration, Alexandria VA, 2003.

ORTIZ-LANDEROS， J.; GÓMEZ-YÁÑEZ， C.; PFEIFFER， H.. Surfactant-assisted hydrothermal crystallization of nano structured lithium metasilicate $\left(\mathrm{Li}_{2} \mathrm{SiO}_{3}\right)$ hollow spheres: II-Textural analysis and $\mathrm{CO}_{2}-\mathrm{H}_{2} \mathrm{O}$ sorption evaluation. J. Solid State Chem., v. 184, p. 22572262. 2011.

ORTIZ-LANDEROS, J.; ÁVALOS-RENDÓN, T. L.; GÓMEZ-YÁÑEZ, C.; PFEIFFER, H. Analysis and perspectives concerning $\mathrm{CO}_{2}$ chemisorption on lithium ceramics using thermal analysis. J. Therm. Anal. Calorim., v. 108, p. 647-655, 2012.

PANNOCCHIA, G.; PUCCINI, M.; SEGGIANI, M.; VITOLO, S. Experimental and modeling studies on high-temperature capture of $\mathrm{CO}_{2}$ using lithium zirconate based sorbents. Ind. Eng. Chem. Res., v. 46, p. 6696-6706. 2007. 
PFEIFFER, H.; BOSCH, P.; BULBULIAN, S. Synthesis of lithium silicates. J. Nucl. Mater., v. 257, p. 309-317. 1998.

SEGGIANI, M.; PUCCINI, M.; VITOLO, S.. High- temperature and low concentration $\mathrm{CO}_{2}$ sorption on $\mathrm{Li}_{4} \mathrm{SiO}_{4}$ based sorbents: Study of the used silica and doping method effects. Inter. J. Greenhouse Gas Control, v. 5, p. 741-748. 2011.

VENEGAS, M. J.; FREGOSO-ISRAEL, E.; ESCAMILLA, R.; PFEIFFER, H. Kinetic and reaction mechanism of $\mathrm{CO}_{2}$ sorption on $\mathrm{Li}_{4} \mathrm{SiO}_{4}$ : Study of the particle size effect. Ind. Eng. Chem. Res, v. 46, p. 2407-2412. 2007. 